\title{
Principaux insectes nuisibles à l'anacardier en Amazonie péruvienne. Données préliminaires
}

\author{
Cesar Delgado ${ }^{1 *}$, Guy CouturieR ${ }^{2}$
}

${ }^{1}$ Inst. Investig. Amazon. Peru.,
Programa investig.
biodiversidad, Apartado 784,
Avenida Abelardo Quiñones,
lquitos, Perú,
cdelgado8@hotmail.com
2 Mus. Natl. Hist. Nat. and
Inst. Rech. Dév., case 50,
57 rue Cuvier, 75231, Paris,
Cedex 05, France
guy.couturier4@wanadoo.fr

${ }^{*}$ Correspondence and reprints

Received 23 October 2013 Accepted 17 January 2014

Fruits, 2014, vol. 69, p. 293-302 (C) 2014 Cirad/EDP Sciences All rights reserved

DOI: 10.1051/fruits/2014018

www.fruits-journal.org

RESUMEN EsPañol, p. 302

\section{Major pests of cashew in the Peruvian Amazon. Preliminary data.}

Abstract - Introduction. The cashew (Anacardium occidentale L.) is mainly grown for the production of cashew nuts, but also for the pseudofruit. The insect pests of the plant were studied in Peru, where this fruit is consumed. Materials and methods. Samples were taken from 1998 to 2005 in different localities of the department of Loreto in the Peruvian Amazon to identify insect pests of cashew. Field observations and laboratory farms were used to characterize the damage caused by each insect. Results. Seven species of insects cause significant damage: five species attack the pseudofruit: Acanthocephala parensis (Hemiptera: Coreidae), Anastrepha obliqua (Diptera: Tephritidae), Conotrachelus sp. (Coleoptera: Curculionidae), Trigona amalthea and T. amazonensis (Hymenoptera: Apidae). The pest Aphis gossypii (Hemiptera: Aphididae) infests the inflorescences, buds and young fruit. The larvae of Macrocopturus cf. tristis (Coleoptera: Curculionidae) develop in buds and young shoots. Three new species are pests which have not yet been reported. Conclusion. Given the potential of the cashew apple production and the possible development of the culture of the cashew tree in the Peruvian Amazon, a more extensive study of the insect pests is desirable. Amazon / Peru / Anacardium occidentale / Anacardiaceae / pests of plants / data
collection / pseudofruits

\section{Principaux insectes nuisibles à l'anacardier en Amazonie péruvienne. Données préliminaires.}

Résumé - Introduction. L'anacardier (Anacardium occidentale L.) est surtout cultivé pour la production de l'amande ou noix de cajou mais aussi pour le pseudofruit. Les insectes nuisibles à cette plante ont été étudiés au Pérou où celui-ci est consommé. Matériel et méthodes. Des prélèvements ont été réalisés de 1998 à 2005 dans différentes localités du département de Loreto en Amazonie péruvienne afin d'identifier les insectes ravageurs de l'anarcadier. Des observations de terrain et des élevages au laboratoire ont permis de caractériser leurs dégâts et des éléments de la bio-écologie pour chacun de ces insectes. Résultats. Sept espèces d'insectes ont été identifiées, qui commettent des dommages notables : cinq espèces s'attaquent au pseudofruit : Acanthocephala parensis (Hemiptera : Coreidae), Anastrepha obliqua (Diptera : Tephritidae), Conotrachelus sp. (Coleoptera : Curculionidae), Trigona amalthea et T. amazonensis (Hymenoptera: Apidae). L'espèce Aphis gossypii (Hemiptera : Aphididae) infeste l'inflorescence ainsi que le fruit. Les larves de Macrocopturus cf. tristis (Coleoptera: Curculionidae) se développent dans les bourgeons et les jeunes rameaux. Trois espèces parmi celles qui ont été identifiées sont de nouveaux ravageurs qui n'avaient pas été encore signalés. Sept espèces d'importance secondaire ont également été observées. Conclusion. Compte-tenu du potentiel que représente la production de pomme cajou et du développement possible de la culture de l'anacardier en Amazonie péruvienne, une étude plus approfondie des insectes nuisibles à cette production est souhaitable.

Amazonie / Pérou / Anacardium occidentale / Anacardiaceae / Ravageur des plantes / collecte de données / pseudofruit

Article published by EDP Sciences 


\section{Introduction}

Le cajou (Anacardium occidentale L. : Anacardiaceae) est un petit arbre natif de la côte nord du Brésil. En raison de son intérêt commercial il est maintenant cultivé au Vietnam, en Inde, au Brésil, au nord de l'Australie, à Hawai, aux îles Fidji et au sud de la Floride principalement [1-3], ainsi que dans différents pays d'Afrique: Nigeria, Tanzanie, Côte d'Ivoire, Guinée-Bissau, Mozambique et Bénin [4]. C'est une espèce qui peut s'adapter à un large spectre écologique [5]. Au Pérou, Anacardium occidentale a surtout une importance locale et c'est la pulpe du pédoncule hypertrophié du fruit, ou pseudofruit, qui est consommée sous forme de jus de fruits, glaces et sorbets.

Pour l'agro-industrie mondiale c'est l'amande ou noix de cajou qui présente actuellement le plus d'intérêt. Selon la FAO, le marché des noix de cajou s'est considérablement développé, les pays asiatiques étant les plus importants producteurs [6]. En 2004, le Vietnam occupait la première place avec $640.000 \mathrm{t}$, suivi de l'Inde avec 460.000 t, du Brésil avec 223.941 t et du Nigéria avec $186.000 \mathrm{t}$.

En Amérique du sud, c'est principalement le Brésil qui a développé des technologies de production de cette culture et plus particulièrement vis-à-vis des ravageurs [7-10] Bien que la noix de cajou soit l'enjeu principal de cette culture, plusieurs auteurs [2, 4, 11-13] font valoir l'intérêt du pseudofruit dont la teneur en vitamine $\mathrm{C}$ est environ 5 fois supérieure à celle de l'orange, soit (200 à 300) mg.100 $\mathrm{g}^{-1}$ [2]. Sous utilisé, trop souvent considéré comme sous produit ou même délaissé, le pseudofruit de l'anacardier peut être utilisé sous forme de jus, sorbets, confiserie, boisson fermentée ainsi que pour l'industrie des cosmétiques. Au Pérou, celui-ci est fréquemment vendu dans les supermarchés et sur les marchés locaux.

Nous avons inventorié les principaux insectes nuisibles à l'anacardier observés en Amazonie péruvienne, et étudié certains éléments sur leur biologie et leur écologie, nécessaires pour mettre en place des méthodes de contrôle intégré de $A$. occidentale.

\section{Matériel et méthodes}

Notre étude a été réalisée entre 1998 et 2005 à l'Instituto de investigaciones de la Amazonia Peruana (IIAP, Pérou) dans ses centres de recherche de Quistococha et Allpahuayo (034' S, $73^{\circ} 16^{\prime} \mathrm{O}$ ) ainsi que dans les communautés de Varillal (0352' S, 7320' O), Peña Negra (03⒌ S, $\left.73^{\circ} 20^{\prime} \mathrm{O}\right)$, Nauta (04³4' S, 7332' O) et Jenaro Herrera (0454' S, 7340' O). L'altitude moyenne de ces localités est de $145 \mathrm{~m}$ au dessus du niveau de la mer. Elle a été réalisée de façon ponctuelle, sans protocole particulier, en fonction de nos possibilités de déplacement et ne prétend pas être exhaustive. Les prélèvements ont été réalisés au hasard en révisant à vue les arbres accessibles dans les vergers (figure 1). Les insectes ravageurs ont été collectés manuellement à différents stades de développement sur les différentes parties des arbres ; ils ont été ensuite transportés au laboratoire pour élevage et obtention des adultes. Les larves ont été élevées dans des cages en plastique aérées et révisées quotidiennement pour changer l'alimentation et nettoyer les cages.

Nous n'avons pas pris en compte les parasitoïdes. Des observations de terrain et au laboratoire ont permis d'obtenir des informations sur la biologie, l'écologie et le type de dégâts.

Le matériel biologique collecté a été déposé dans les collections de l'Instituto de Investigaciones de la Amazonía Peruana à Iquitos (Pérou) et celles du Muséum national d'Histoire naturelle de Paris (France).

\section{Résultats}

Durant notre étude nous avons observé sept ravageurs qui commettent des dégâts d'importance majeure ou pouvant nuire à la productivité. Parmi eux, cinq attaquent le pseudofruit : Acanthocephala parensis, Anastrepha obliqua, Conotrachelus sp., Trigona amalthea et T. amazonensis; le sixième, Aphis gossypii, infeste l'extrémité des rameaux, les inflorescences et les jeunes pseudofruits et le septième, Macropturus cf. tristis, fore les rameaux. Sept autres ravageurs identifiés produisent des dégâts moins 
importants : Gymnetis pantherina ssp. meleagris Burmeister, Hoplopyga liturata Olivier, Megalopyge lanata Stoll, Membracis sp., Oncideres crassicornis Bates, Periphoba hircia Hübner et Zoreva sp.

\subsection{Ravageurs d'importance majeure}

\subsubsection{Acanthocephala parensis (Dallas) (Hemiptera : Coreidae)}

Acanthocephala parensis est une punaise de grande taille, environ $22 \mathrm{~mm}$ de long, de couleur noire. Ses fémurs postérieurs présentent une série de 5 à 6 "dents "fortes ; les tibias postérieurs montrent une expansion latérale aplatie sur toute leur longueur, ainsi qu'une denticulation fine chez le mâle ; le tarse est marron clair. Les jeunes et adultes s'alimentent du pseudofruit vert ou en processus de maturité (figure 2). Si le fruit attaqué est jeune, il se dessèche et noircit. À un état de développement plus avancé du pseudofruit, celui-ci prend une coloration sombre et il est attaqué par divers champignons. Acanthocephala parensis a aussi été observé sur le fruit. Cette espèce est signalée pour la première fois sur l'anacardier. En Amazonie péruvienne, nous l'avons observé dans les stations de Quistococha, el Varillal et Jenaro Herrera.

\subsubsection{Anastrepha obliqua Macquart (Diptera : Tephritidae)}

Anastrepha obliqua est une mouche des fruits mesurant $7 \mathrm{~mm}$ de long ; les ailes sont transparentes avec de larges bandes brunjaune légèrement brunies (figure 3). C'est une espèce polyphage largement distribuée dans toute l'Amazonie et la zone néotropicale. Les femelles perforent l'épiderme du pseudofruit avec leur ovipositeur et y insèrent leurs œufs. Les larves se développent dans la pulpe du pseudofruit ; à leur complet développement elles mesurent $8 \mathrm{~mm}$ de longueur. Leur développement terminé les larves se laissent tomber au sol où elles s'empupent à faible profondeur. Ce sont des asticots qui ne doivent pas être confondues avec les larves de Conotrachelus sp. (voir ci-dessous). Comme pour ce dernier, le ramassage et la destruction des pseudofruits

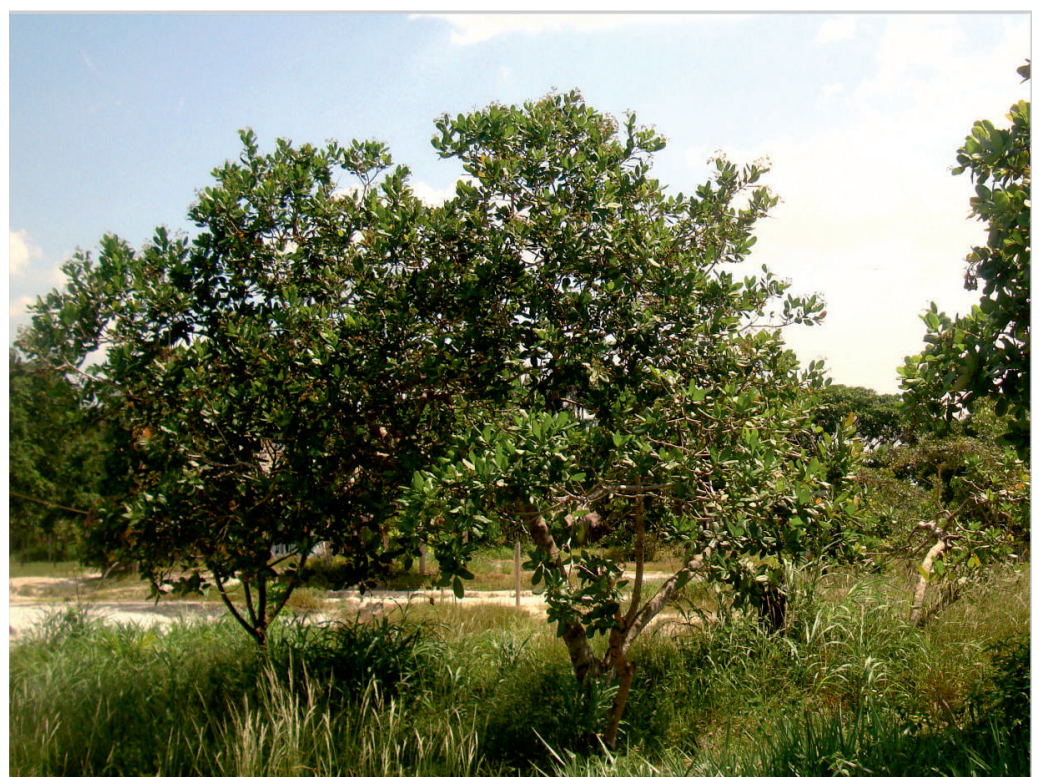

Figure 1.

Anacardiers aux abords du village de Varillal (Pérou).

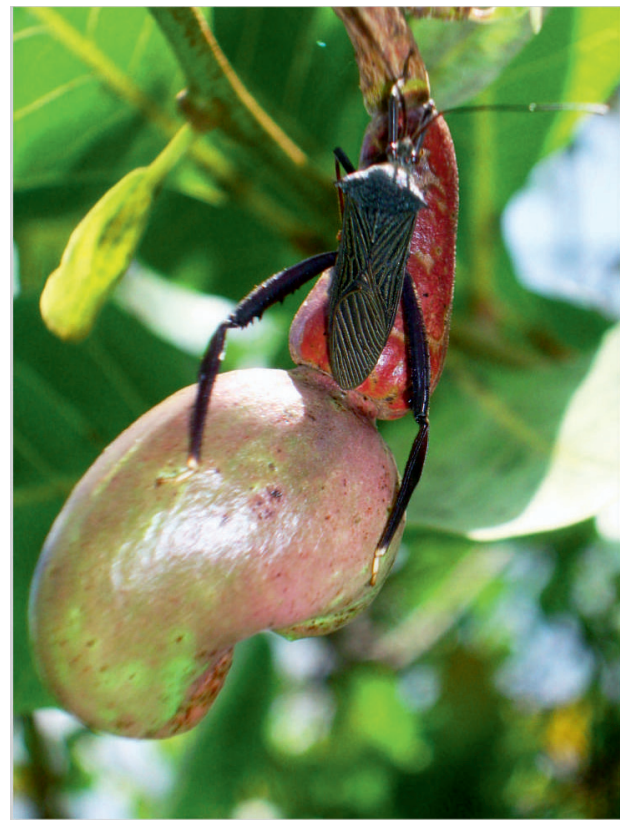

infestés sont recommandés. En Amazonie péruvienne plusieurs espèces de mouches des fruits ont été citées sur d'autres arbres fruitiers où elles occasionnent des dégâts notables [14, 15-17]. Anastrepha obliqua est largement distribuée dans toute l'Amazonie. Sur l'anacardier elle a été observée
Figure 2.

Acanthocephala parensis sur un pseudofruit d'anacardier. 


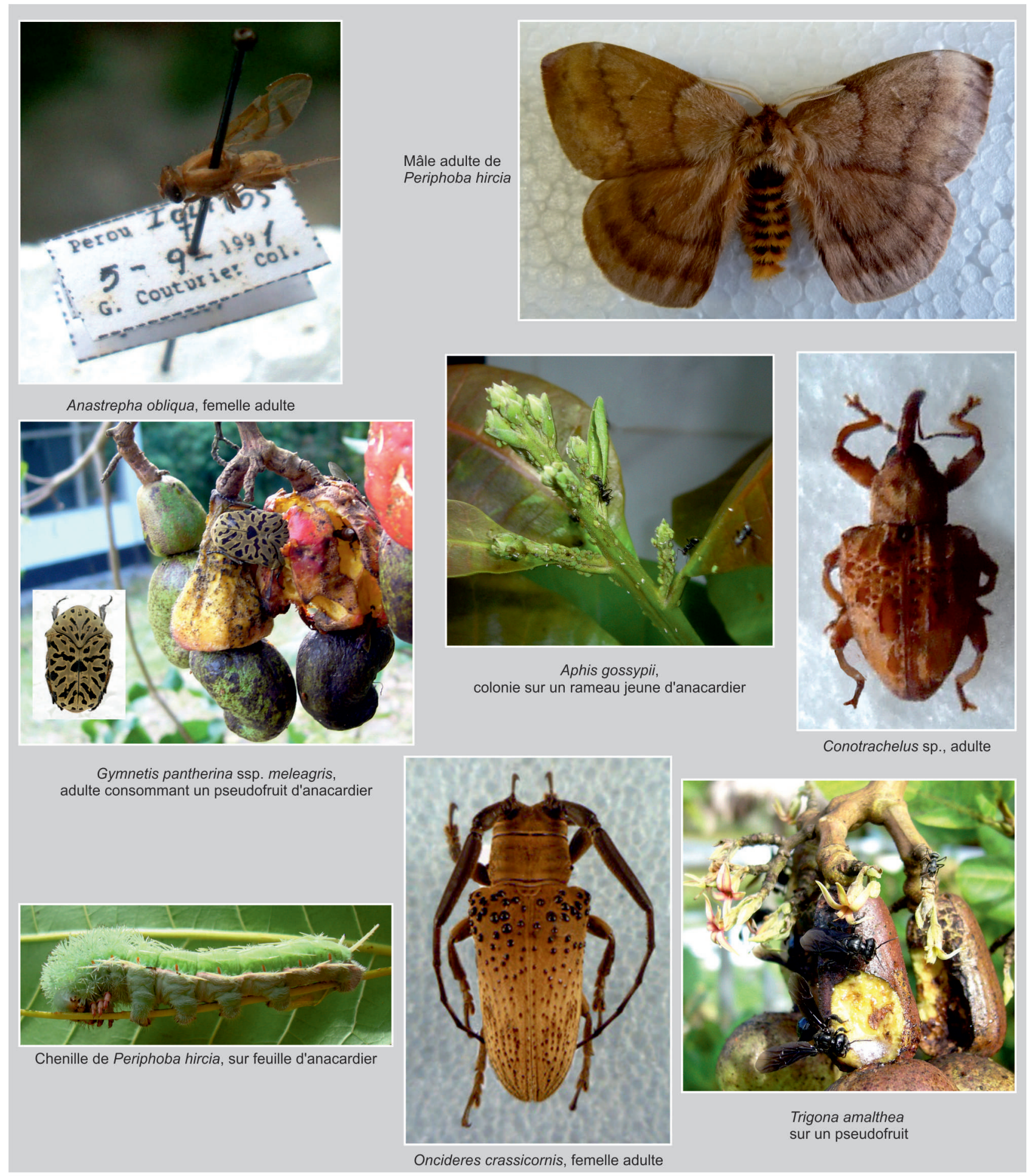

Figure 3.

Divers ravageurs provoquant des dégâts plus ou moins importants sur anacardier au Pérou. 
à Iquitos et à Pucallpa, où elle cause des dégâts considérables.

\subsubsection{Conotrachelus sp. (Coleoptera : Curculionidae)}

Le genre Conotrachelus comprend de très nombreuses espèces dont beaucoup sont nuisibles aux cultures fruitières. Elles sont le plus souvent spécifiques d'une espèce ou d'un genre végétal. L'espèce qui s'attaque au pseudofruit de l'anacardier n'est pas décrite ; elle est considérée comme nouvelle pour la science (C.W. O'Brien, commun. pers.). La larve est de couleur blanccrème, à tête marron ; elle s'alimente de la pulpe du pseudofruit qui peut être totalement détruite ; celui-ci durcit et se nécrose. À la fin de son développement larvaire, la larve abandonne le fruit se laisse tomber au sol où elle s'enterre à faible profondeur. La nymphose dure 6 à 8 jours. L'adulte mesure $5 \mathrm{~mm}$ de long et le rostre fait $1,5 \mathrm{~mm}$. De couleur marron à marron clair, le pronotum et les élytres sont recouverts de protubérances caractéristiques (figure 3). Durant le jour les adultes restent au sol, dans la végétation ou dans les débris accumulés sur les branches. Au crépuscule ils montent sur le tronc, s'accouplent et pondent sur le pseudofruit. Au cours de notre étude l'espèce a été observée dans les stations de Quistococha, Allpahuayo et Jenaro Herrera.

\subsubsection{Trigona amalthea (Olivier)} et $T$. amazonensis Ducke (Hymenoptera : Apidae)

Trigona amalthea (Olivier) et T. amazonensis Ducke sont deux abeilles de couleur noire de 6 à $7 \mathrm{~mm}$ de long, sans aiguillon ; leurs ailes sont sombres, bleutées, plus larges que l'abdomen. Ces espèces vivent dans des nids contenant de très nombreux individus. Les adultes s'alimentent du pseudofruit à tous les stades de son développement (figure 3). Sur le pédoncule mûr elles peuvent creuser de profondes cavités pour en extraire la pulpe; ces cavités servent d'accès à d'autres insectes détritivores qui favorisent ainsi la décomposition du pseudo fruit. Si ces abeilles étaient trop abondantes, la lutte consisterait à les détruire après localisation de leurs nids.

\subsubsection{Aphis gossypii Glover (Homoptera : Aphididae)}

Aphis gossypii Glover est le "puceron du cotonnier ", insecte de couleur jaune clair à vert sombre, mesurant jusqu'à $1,8 \mathrm{~mm}$ de long. Il vit en colonies denses, suce la sève des bourgeons, inflorescences et fruits en formation (figure 3). Il s'ensuit un dessèchement des fleurs et bourgeons qui provoquent des malformations sur les pseudofruits. Les sécrétions sucrées émises par ces insectes (miellat) favorisent la présence de fumagine qui nuit à leur développement et c'est la présence de fourmis (non identifiées) qui, en consommant ces sécrétions, favorise leurs pullulations en les protégeant des prédateurs. Différents insectes prédateurs (coléoptères Coccinellidae, larves de diptères Syrphidae) limitent cependant ces pullulations. La production de l'anarcadier peut être affectée : Melo et al. indiquent que jusqu'à $76 \%$ d'inflorescences peuvent être détruites [18]. Bleicher et al. ont observé que les pertes de production peuvent atteindre 36,8 \% [19]. Aphis gossypii est une espèce cosmopolite, polyphage, connue sur de nombreux hôtes appartenant à différentes familles végétales en Amérique du sud [10, 20-23]. Dans le cadre de notre étude, l'insecte a été observé dans les stations de Quistococha, Nauta et Jenaro Herrera ; il a été récemment rapporté sur une myrtacée locale, Myrciaria dubia [24].

\subsubsection{Macrocopturus cf. tristis (Heller) (Coleoptera : Curculionidae)}

L'adulte de Macrocopturus $\mathrm{cf}$. tristis (Heller) est un charançon de couleur noir-brillant de $5 \mathrm{~mm}$ de long ; il montre un rostre long, replié au repos sur la face ventrale et atteignant l'abdomen (figure 4). La larve est de couleur blanc-crème ; la tête est marron ; la larve mesure 6 à $7 \mathrm{~mm}$ de long. La femelle adulte dépose ses oufs dans le bourgeon terminal d'une branche d'anacardier. La larve pénètre dans celui-ci et fore une galerie descendante dans le rameau où elle s'alimente pendant plusieurs mois. Il peut y avoir plusieurs larves sur une même branche mais seulement une seule larve par galerie. La plante exsude une abondante résine gommeuse, transparente, qui durcit et prend une coloration marron (figure 5) ; 


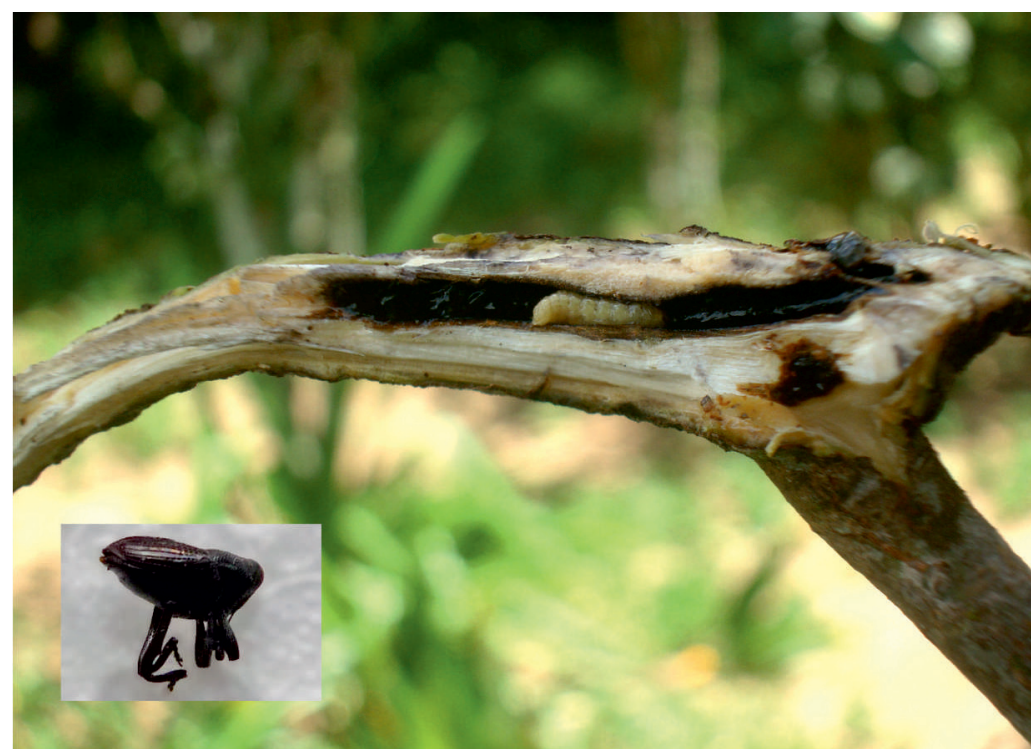

Figure 4.

Macrocopturus cf. tristis, galerie ouverte et insecte adulte sur anacardier.

\section{Figure 5.}

Exsudation de sève sur anacardier, due aux attaques de Macrocopturus cf. tristis. la première fois au centre scientifique de Quistococha puis à Jenaro Herrera et dans la localité de Varillal.

\subsection{Ravageurs d'importance secondaire}

\subsubsection{Gymnetis pantherina ssp. meleagris Burmeister et Hoplopyga liturata Olivier (Coleoptera : Cetoniidae)}

Les adultes des deux espèces Gymnetispantherina ssp. meleagris Burmeister et Hoplopyga liturata Olivier s'attaquent aux pseudofruits mûrs ou en début de maturité ; ils s'alimentent de la pulpe, causant des lésions importantes et rendant le fruit impropre à la consommation. Gymnetis pantherina ssp. meleagris (figure 3) est de couleur brunverdâtre avec de nombreuses taches noires sur le pronotum et les élytres; il mesure $20 \mathrm{~mm}$ de long. Hoplopyga liturata est plus petit (17 mm de long) et les taches sont beaucoup plus fines et nombreuses. Nous n'avons pas observé de larves, qui se développent habituellement dans les bois décomposés et les terreaux. Les dégâts dus à ces deux ravageurs sont sporadiques et ne justifient pas d'intervention particulière. Toutefois, les adultes visibles sur les pseudofruits peuvent être prélevés et détruits pour limiter la prolifération de ces espèces.

\subsubsection{Megalopyge lanata Stoll (Lepidoptera : Megalopygidae)}

La chenille de Megalopyge lanata Stoll est défoliatrice; elle est de couleur blanc-bleuté et pourvue de longues soies très urticantes. Polyphage, elle est connue sur 14 familles botaniques différentes mais sa rareté en fait un ravageur très secondaire. L'adulte est de couleur brun clair, variable. Les ailes sont partiellement transparentes. L'incidence des attaques est négligeable.

à l'ouverture la galerie présente une coloration noire caractéristique (figure 4). Les branches sont fragilisées et peuvent casser sous l'effet du vent ou d'une forte charge en fruits. Les dégâts se remarquent facilement par la présence de boules de gomme. Ce ravageur ne semble pas avoir été signalé auparavant. Il a été rencontré pour

\subsubsection{Membracis sp. (Hemiptera : Membracidae)}

Les jeunes ravageurs du genre Membracis sont de couleur blanche ; ils peuvent recouvrir presque entièrement le fruit (figure O) et nuisent à son développement. Les adultes 
mesurent $8 \mathrm{~mm}$ de long ; ils sont de couleur noire, tachés de blanc, aplatis latéralement et leur pronotum, en forme de lame, recouvre l'ensemble de l'abdomen. Leurs dégâts sont limités en raison du faible nombre de pseudofruits attaqués peuvant être détruits.

\subsubsection{Oncideres crassicornis Bates (Coleoptera : Cerambycidae)}

Les femelles de Oncideres crassicornis Bates (figure 3), ravageur longicorne, pondent dans les branches de l'anacardier qu'elles découpent ensuite. Les larves se développent en plusieurs semaines ou plusieurs mois. L'espèce est polyphage. Les dégâts sont le plus souvent très localisés et peu importants. Il est préconisé de détruire les branches coupées qui contiennent les larves.

\subsubsection{Periphoba hircia Hübner (Lepidoptera : Saturniidae)}

Les chenilles de Periphoba hircia Hübner s'alimentent du feuillage ; elles mesurent de 5 à $6 \mathrm{~cm}$ en fin de développement, sont de couleur verte et présentent des soies urticantes blanches (figure 3). Lorsqu'elles sont nombreuses, elles peuvent défolier les arbres de façon notable. Le papillon adulte est de couleur marron uniforme excepté, sur les ailes postérieures, deux lignes courbes, concentriques plus ou moins en zigzag, plus foncées que le fond de l'aile et, sur les ailes antérieures, trois lignes de même aspect (figure 3). L'espèce a été observée dans la station d'el Varrillal. Ravageur secondaire, Periphoba hircia devra cependant être prise en considération en cas de pullulation. Dans ce cas il conviendra d'éliminer les chenilles.

\subsubsection{Zoreva sp. (Hemiptera : Coreidae)}

Larves et adultes de Zoreva sp. se rencontrent sur les pseudofruits où ils provoquent des nécroses. C'est une punaise de $15 \mathrm{~mm}$ de long de couleur jaune et brun-noir (figure 7). Cette espèce est rare sur l'anacardier et ne justifie pas d'intervention particulière.

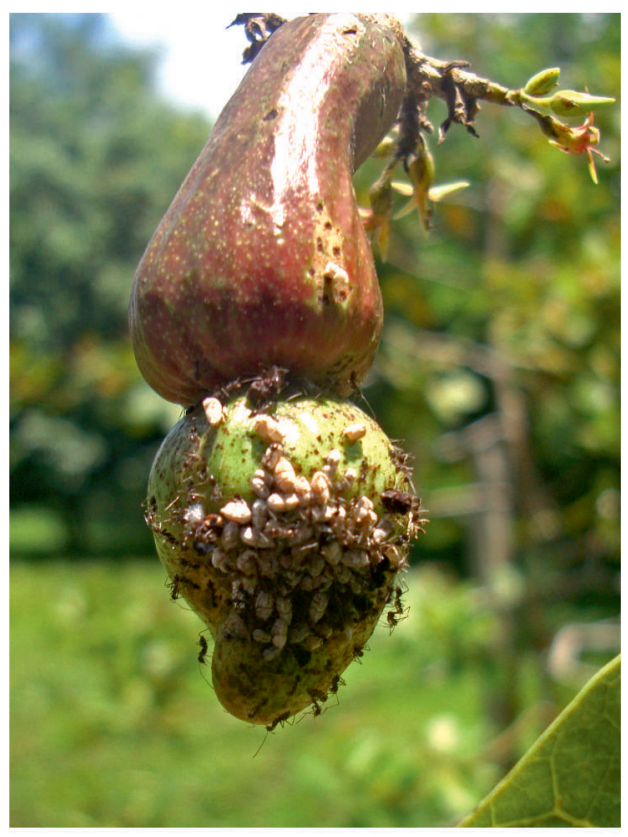

Figure 6.

Jeunes de Membracis sp. sur fruit d'anacardier.

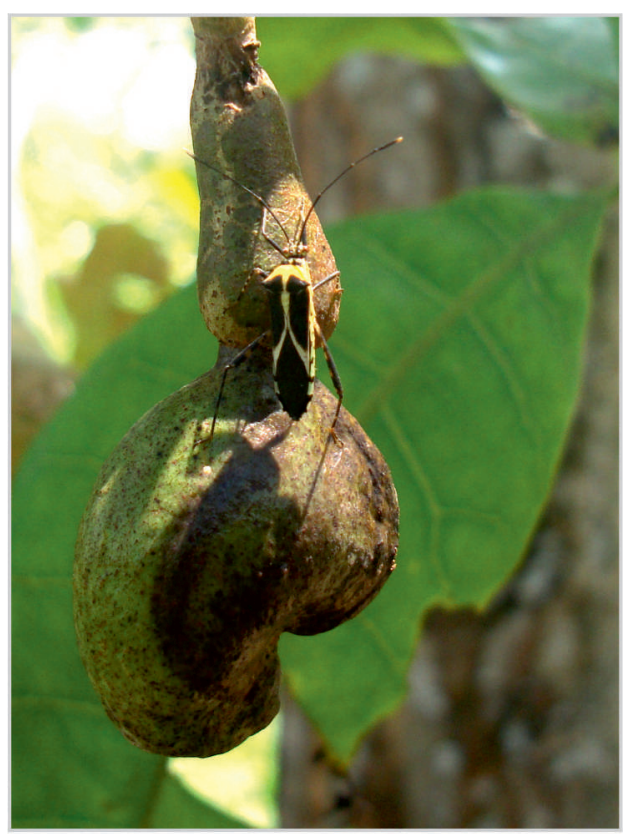

Figure 7.

Adulte de Zoreva sp. piquant un pseudofruit d'anacardier.

\section{Discussion et conclusion}

Au Pérou, la production de noix de cajou pour la consommation locale et l'exportation est négligeable. Le pseudofruit, cependant, est localement très consommé et, en Amazonie, il provient de petits vergers plus 
Table I.

Insectes nuisibles à l'anacardier observés en Amazonie péruvienne de 1998 à 2005.

\begin{tabular}{|c|c|c|c|}
\hline Genre, espèce & Ordre, famille & Partie attaquée & Incidence \\
\hline \multicolumn{4}{|l|}{ Ravageurs importants } \\
\hline Acanthocephala parensis & Hemiptera : Coreidae & Pseudofruit & Élevée $^{1}$ \\
\hline Anastrepha obliqua & Diptera : Tephritidae & Pseudofruit & Élevée ${ }^{1}$ \\
\hline Aphis gossypii & Hemiptera : Aphididae & Extrémité des rameaux, bourgeons & Faible \\
\hline Conotrachelus sp. & Coleoptera : Curculionidae & Pseudofruit & Élevée $^{1}$ \\
\hline Macropturus cf. tristis & Coleoptera : Curculionidae & Rameaux, bourgeons & Élevée $^{1}$ \\
\hline $\begin{array}{l}\text { Trigona amalthea, } \\
\text { T. amazonensis }\end{array}$ & Hymenoptera : Apidae & Pseudofruit & Élevée ${ }^{1}$ \\
\hline \multicolumn{4}{|l|}{ Ravageurs secondaires } \\
\hline $\begin{array}{l}\text { Gymnetis pantherina } \\
\text { Hoplopyga liturata }\end{array}$ & Coleoptera : Cetoniidae & Pseudofruit & Faible \\
\hline Megalopyge lanata & Lepidoptera : Megalopygidae & Feuilles & Faible \\
\hline Membracis sp. & Hemiptera : Membracidae & Pseudofruit & Faible \\
\hline Oncideres crassicornis & Coleoptera : Cerambycidae & Branches & Faible \\
\hline Periphoba hircia & Lepidoptera : Saturniidae & Feuilles & Faible \\
\hline Zoreva sp. & Hemiptera : Coreidae & Pseudofruit & Négligeable \\
\hline
\end{tabular}

ou moins bien entretenus ou de plantes isolées. L'anarcardier, d'une culture relativement facile, mériterait d'être planté sur des terres qui parfois sont impropres à des cultures plus exigeantes. En raison du faible nombre d'arbres cultivés en Amazonie péruvienne, la diversité des insectes nuisibles est limitée. Cependant, plusieurs espèces que nous avons identifiées sont considérées comme étant de nouveaux ravageurs et s'ajoutent à la liste de ceux d'Amérique du Sud qui sont déjà connus sur l'anacardier. Plusieurs espèces du genre Conotrachelus ont été rapportées récemment en Amazonie péruvienne sur différentes myrtacées: la goyave (Psidium guajava), l'araza (Eugenia stipitata) et le camu camu (Myrciaria dubia) [15, 17, 24]. Dans l'état d'Amazonas, au Brésil, l'anacardier est cité comme unique plante-hôte d'Anastrepha leptozona $[25,26]$, bien que celle-ci soit connue sur d'autres plante-hôtes. Dans le Nordeste brésilien le papillon Anthistarcha binocularis (Lepidoptera : Gelechiidae) cause des dégâts similaires à ceux de $M$. tristis sur l'anacardier [10]. Les abeilles du genre Trigona sont connues comme ravageurs de différentes plantes fruitières en Amazonie péruvienne [16, 17]. Au Brésil, T. spinipes est citée comme ravageur de l'anacarde [20]. Différentes études réalisées dans d'autres pays producteurs ont montré l'intérêt de la consommation du pseudofruit d'un point de vue nutritionnel ; ils relatent que ce sousproduit est trop souvent négligé. En raison du potentiel représenté par cette espèce pour l'agro-industrie nationale péruvienne ainsi que pour la consommation locale, il apparaît nécessaire d'adapter les techniques et les variétés qui permettraient le développement de cette culture en Amazonie. Cette démarche passe par le contrôle des insectes nuisibles qui sont un facteur limitant pour la production de pseudofruits de qualité. Des méthodes de contrôle intégré devront être mises au point.

\section{Remerciements}

Cette étude a été réalisée dans le cadre de la convention existant entre l'IIAP et l'IRD (Institut de recherche pour le développement) et financée par le projet INCAGRO. Les auteurs remercient les ingénieurs 
A. Gonzales et S. Tello, respectivement directeurs des centres de recherche de Allpahuayo et de Quistococha ; J. Vasquez et $\mathrm{H}$. Pacaya pour leur assistance sur le terrain ; A. Aing (IRD, Service de cartographie) pour le traitement numérique des figures, ainsi que les taxonomistes qui ont identifié les insectes : H. Brailovsky (Coreidae), J.P. Legrand (Cetoniidae), J. Minet (Lepidoptera), C.W. O'Brien (Curculionidae), C. Rassmussen (Apidae), G. Tavakilian (Cerambycidae), R.A. Zucchi (Tephritidae).

\section{Références}

[1] Lima V.P.M.S., A cultura do cajueiro no Nordeste do Brasil. Banco do Nordeste do Brasil, Escrit. Técn. Estud. Econ. Nordeste, Fortaleza, Brazil, 1998, 498 p.

[2] Lautié E., Dornier M., Souza Filho M. de, Reynes M., Les produits de l'anacardier, caractéristiques, voies de valorisation et marché, Fruits 56 (2001) 235-248.

[3] Souza R.P., Ribeiro R.V., Machado E.C., Oliveira R. F., Silveira J.A.G. da, Photosynthetic responses of young cashew plants to varying environmental contitions, Pesq. agropec. Bras. 40 (2005) 735-744.

[4] Anon., Anacarde, une noix en pleine transformation, Spore 123 (2006) 3.

[5] Mendes-Ferrão J.E., O Cajueiro, Inst. Investig. Cient. Trop., Lisboa, Portugal, 1995, $298 \mathrm{p}$.

[6] Paiva J.R., Cavalcanti J.J.V., Barros L.M., Corrêa M.C.M., Maia M.C.C., Costa Filho A.B., Seleção de clones de cajueiro comum pelo método em "tandem" e índice de classificação., Ciênc. Agrotec. 31 (2007) 765772.

[7] Silva A.G., Primeira contribução ao conhecimento biocronológico da ocorrência de pragas no Ceará, Bol. Soc. Cear. Agron. 2 (1961) 47-50.

[8] Bleicher E., Melo Q.M.S., Artrópodes associados ao cajueiro no Brasil, EmbrapaCNPAT, Doc. $n^{\circ}$ 9, Fortaleza, Brazil, 1993, $33 \mathrm{p}$.

[9] Mesquita A.L.M., Becker V.O., Sobrinho R.B., Taxonomic identification of lepidopterous species of cashew plant in Brazil, Ann. Soc. Entomol. Bras. 27 (1998) 655-656.
[10] Melo Q.M.S., Bleicher E., Pragas do cajueiro, in: Sobrinho R.B., Cardoso J.E., Freire F.Ch.O. (Eds.), Pragas de fruteiras Tropicais de importância Agroindustrial, EMBRAPA, Brasília DF, Brazil, 1998.

[11] Tandjiekpon A.M., Caractérisation du système agroforestier à base d'anacardier (Anacardium occidentale Linnaeus) en zone de savane du Bénin, Univ. Abomey-Calavi, Thèse, Benin, 2005, $122 \mathrm{p}$

[12] Sivagurunathan P., Sivasankari S., Muthukkaruppan S.M., Characterisation of cashew apple (Anacardium occidentale L.) fruits collected from Ariyalur District, J. Biosci. Res. 1 (2010) 101-107.

[13] Gordon A., Friedrich M., Martins V. da M., Moura C.F.H., Marx F., Changes in phenolic composition, ascorbic acid and antioxydant capacity in cashew apple (Anacardium occidentale L.) during ripening, Fruits 67 (2012) 267-276.

[14] Couturier G., Zucchi R.A., Saravia G.M., Silva N.M., New records of fruit flies of the genus Anastrepha Schiner, 1868 (Diptera: Tephritidae), and their host plants in the Amazon region, Ann. Soc. Entomol. Fr. (NS), 29 (1993) 223-224.

[15] Couturier G., Quiñones L.R., Gonzáles I.R., Riva R.R., Young F.R., Los insectos plaga de las Myrtaceae frutales en Pucallpa, Amazonía peruana, Rev. Per. Entomol. 39 (1996) 125-130.

[16] Couturier G., Tanchíva E., Gonzalez J., Cardenas R., Inga H., Observations préliminaires sur les insectes nuisibles à l'araza (Eugenia stipitata Mc Vaugh, Myrtaceae) nouvelle culture fruitière, Fruits 51 (1996) 229-239.

[17] Vásquez J., Delgado C., Couturier G., Ferrero D.M., Les insectes nuisibles au goyavier (Psidium guajava L. : Myrtaceae) en Amazonie péruvienne, Fruits 57 (2002) 323-334.

[18] Melo Q.M.S., Bleicher E., Oliveira I.S.R., Couthinho C.I.S.B., Macul F., Efeito de deltamethrin e mancozeb sobre Anthistarcha binocularis (Lep.: Gelechiidae), Selenothrips rubrocinctus (Thysanoptera: Thripidae) e Aphis gossypii (Hom.: Aphididae) em cajueiro, Embrapa-CNPCa, Bol. Pesqu. $n^{\circ} 6$, Fortaleza, Brazil, 1992.

[19] Bleicher E., Abreu A.R.M., Melo Q.M.S., Controle químico do pulgão da inflorescência do cajueiro com produtos seletivos, Embrapa-CNPAT, Bol. Pesqu. $n^{\circ} 12$, Fortaleza, Brazil, 1994. 
[20] Saunders L.J., Coto T.D., King A.B.S., Plagas invertebradas de cultivos anuales alimenticios en America Central, $2^{\circ}$ ed, CATIE, Manual Técn. $\mathrm{n}^{\circ}$ 29, Turrialba, Costa Rica, 1998.

[21] Lima A. da C., Insectos do Brasil, $3^{\circ}$ Tomo, Homópteros, Esc. Nac. Agron., Ser. Didact. $n^{\circ}$ 4, São Paulo, Brazil, 1942, 327 p.

[22] Gallo D., Nakano O., Silveira Neto S., Carvalho R.P.L., Batista G.C., Berti Filho E., Parra J.R.P., Zucchi R.A., Alves S.B., Vendramim J.D., Manual de entomologia agricola, Agron. Ceres, São Paulo, Brazil, 1988.

[23] Couturier G., Tanchiva E.F., Cardenas R.M. Gonzales T.J., Inga H., Los insectos plagas del camu-camu (Myrciaria dubia H.B.K.) y el araza (Eugenia stipitata Mc. Vaugh). Identificación y Control, INIA, Ser. Inf. Técn. $n^{\circ} 26$, Lima, Peru, 1994.

[24] Delgado C., Couturier G., Manejo de insectos plagas en la Amazonía: Su aplicación en camu camu, IIAP-IRD, Lima, Perú, 2004.

[25] Silva N.M. da, Ronchi-Teles B., Moscas-dasfrutas dos Estados brasileiros: Amapá, Amazonas, Pará, Rondônia e Roraima, in: Malavasi A., Zucchi R. (Eds.), Moscas-dasfrutas de importância econômica no Brasil, Holos, São Paulo, Brazil, 2000.

[26] Zucchi R., Taxonomia, in: Malavasi A., Zucchi R. (Eds.), Moscas-das-frutas de importancia econômica no Brasil, Holos, São Paulo, Brazil, 2000.

\section{Principales insectos plagas del marañón en la Amazonía peruana. Datos preliminares.}

Resumen - Introducción. El anacardo (Anacardium occidentale L.) se cultiva principalmente para la producción de almendras o castañas de cajú, sino también para el pseudofruto. Los insectos plagas de la planta fueron estudiados en el Perú, donde se consume. Materiales y métodos. De 1998 a 2005 se tomaron muestras en diferentes localidades del departamento de Loreto en la Amazonía peruana para identificar las plagas de los anacardos. Las observaciones de campo y de laboratorio se utilizaron para caracterizar el daño a cada uno. Resultados. Siete especies de insectos cometen un daño significativo: cinco atacan el pseudofruto: Acanthocephala parensis (Hemiptera: Coreidae), Anastrepha obliqua (Diptera: Tephritidae), Conotrachelus sp. (Coleoptera: Curculionidae) Trigona amalthea y T. amazonensis (Hymenoptera: Apidae). Aphis gossypii (Hemiptera: Aphididae) infesta la inflorescencia, el fruto joven y los brotes. Las larvas de Macrocopturus cf. tristis (Coleoptera: Curculionidae) se desarrollan en los brotes y ramas jóvenes. Tres especies son nuevos reportes para el cultivo. Conclusión. Dado el potencial de la producción de manzanas de marañón y el posible desarrollo de la cultura del anacardo en la Amazonía peruana, un estudio más profundizado de los insectos plagas es deseable.

Amazonia / Perú / Anacardium occidentale / Anacardiaceae / Plagas de plantas / colección de datos / pseudofruto 